\title{
Normal pressure hydrocephalus due to an iatrogenic giant lumbar pseudomeningocele after posterior lumbar interbody fusion: illustrative case
}

\author{
Yoshiomi Kobayashi, MD, PhD, Kanehiro Fujiyoshi, MD, PhD, Toshiyuki Shimizu, MD, Yosuke Kawano, MD, Toshiki Okubo, MD, PhD, \\ Yoshihide Yanai, MD, Takashi Kato, MD, Kohei Matsubayashi, MD, PhD, Keitaro Matsukawa, MD, PhD, Mitsuru Furukawa, MD, PhD, \\ Tsunehiko Konomi, MD, PhD, Junichi Yamane, MD, PhD, Masakazu Takemitsu, MD, PhD, and Yoshiyuki Yato, MD, PhD
}

Department of Orthopedic Surgery, National Hospital Organization Murayama Medical Center, Tokyo, Japan

BACKGROUND latrogenic pseudomeningocele incidence after lumbar surgery is $0.068 \%-2 \%$, and most lumbar pseudomeningoceles are smaller than $5 \mathrm{~cm}$; however, in rare cases, "giant" pseudomeningoceles greater than $8 \mathrm{~cm}$ in size may develop. Normal pressure hydrocephalus (NPH) is another rare condition in which the ventricles expand despite the presence of normal intracranial pressure. To date, pseudomeningocele associated with NPH has not been reported.

OBSERVATIONS An 80-year-old woman underwent L3-5 laminectomy and posterior lumbar interbody fusion, and her symptoms improved after surgery. However, dementia appeared 1 month after surgery. Repeated brain computed tomography showed ventricular enlargement, and lumbar magnetic resonance imaging showed a long pseudomeningocele in the subcutaneous tissues at the L4 level. Here, the authors report a rare case of an iatrogenic giant pseudomeningocele accompanied by NPH after lumbar surgery. The symptoms of NPH in the present case occurred after spinal surgery and recovered after dural repair surgery, indicating that the changes in cerebrospinal fluid circulation and/or pressure due to pseudomeningoceles may cause NPH.

LESSONS The prevention of dural tears through precise surgical technique and primary repair of dural tears are the best approaches to prevent pseudomeningocele incidence and subsequent events.

https://thejns.org/doi/abs/10.3171/CASE224

KEYWORDS giant lumbar pseudomeningocele; normal pressure hydrocephalus; dural tear; posterior lumbar interbody fusion; spine surgery; ventriculoperitoneal shunt

Pseudomeningocele is defined as an extradural abnormal cystic collection of cerebrospinal fluid (CSF) without the dura mater that results after a rupture in the dural arachnoid layer. ${ }^{1}$ The etiology can be iatrogenic, traumatic, or congenital. ${ }^{2,3}$ latrogenic pseudomeningocele incidence after lumbar surgery is $0.068 \%-2 \%$, where the pseudomeningoceles are often small, asymptomatic, and spontaneously disappearing. ${ }^{5}$ However, some patients may present with low back pain and neurological symptoms, including radiculopathy, headache, or local swelling. Most lumbar pseudomeningoceles are smaller than $5 \mathrm{~cm}$; however, in rare cases, "giant" pseudomeningoceles greater than $8 \mathrm{~cm}$ in size may develop. ${ }^{1,3}$

Normal pressure hydrocephalus (NPH) is another rare condition in which the ventricles expand despite the presence of normal intracranial pressure. It presents with the classic triad of dementia, gait disturbance, and bladder disorder. ${ }^{6}$ Furthermore, the etiology is usually idiopathic but can be associated with previous intracranial hemorrhage, head injury, or meningitis. To date, pseudomeningocele associated with NPH has not been reported. Here, we report a rare case of an iatrogenic giant pseudomeningocele accompanied by NPH after lumbar surgery.

\section{Illustrative Case}

An 80-year-old woman with a 1-year history of low back pain and radiculopathy in both legs was referred to our hospital after magnetic resonance imaging (MRI) detected lumbar spinal stenosis.

ABBREVIATIONS CSF = cerebrospinal fluid; $\mathrm{CT}=$ computed tomography; MRI = magnetic resonance imaging; $\mathrm{NPH}=$ normal pressure hydrocephalus; $V P=$ ventriculoperitoneal.

INCLUDE WHEN CITING Published March 7, 2022; DOI: 10.3171/CASE224.

SUBMITTED January 4, 2022. ACCEPTED January 20, 2022.

(c) 2022 The authors, CC BY-NC-ND 4.0 (http://creativecommons.org/licenses/by-nc-nd/4.0/). 


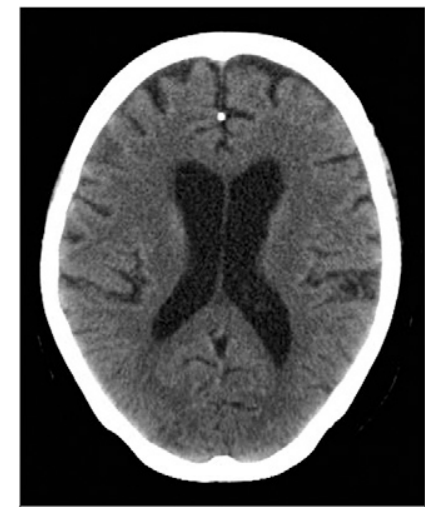

1 month after surgery

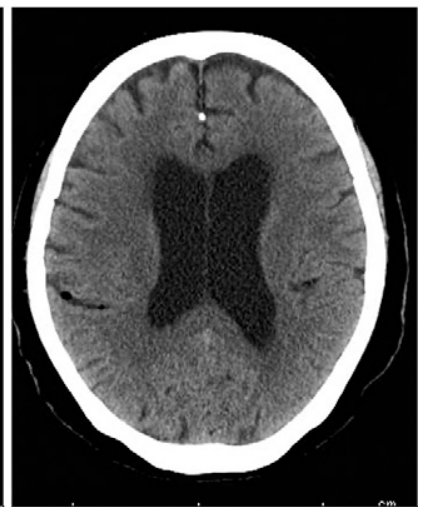

2 months after surgery
FIG. 1. Brain CT after spine surgery. Left: Brain CT showed no significant findings at 1 month after lumbar surgery. Right: Brain CT demonstrated ventricular enlargement but no brain hemorrhage or infarction at 2 months after lumbar surgery.

Her neurological examination revealed mild sensory deficit in both legs along with hypoexcitability of the patellar tendon reflex on the right side. No bladder or bowel dysfunction was observed. An L3-5 laminectomy and posterior lumbar interbody fusion were performed. After surgery, the patient's radiculopathy improved, and walking rehabilitation was initiated. Approximately 1 month after surgery, dementia appeared; however, no significant results were found on brain computed tomography (CT) (Fig. 1, left). At 2 months after surgery, the patient experienced urinary dysfunction and difficulty in gait. Repeated brain CT showed ventricular enlargement but no brain hemorrhage or infarction (Fig. 1, right).

Lumbar MRI revealed a small tear within the posterior dura at level L4 and a substantially sized collection of CSF in a long pseudomeningocele in the subcutaneous tissues, measuring $25 \mathrm{~cm}$ in length (Fig. 2, left) with a maximum cross-sectional size of $11 \times 4 \mathrm{~cm}$ (Fig. 2, right).

The patient underwent excision of the pseudomeningocele and dural repair. A posterior CSF fistula without nerve root entrapment was
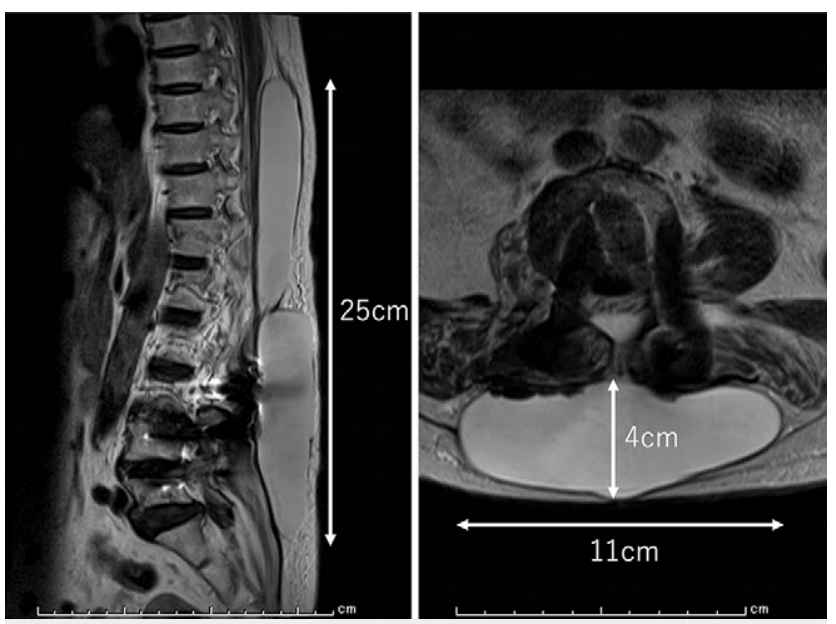

FIG. 2. Lumbar MRI after spine surgery. Left: T2-weighted sagittal image. Right: T2-weighted axial image. A small tear within the posterior dura at level L4 and a substantially sized collection of CSF in a long pseudomeningocele in the subcutaneous tissues, measuring $25 \mathrm{~cm}$ in length (left) with a maximum cross-sectional size of $11 \times 4 \mathrm{~cm}$ (right).

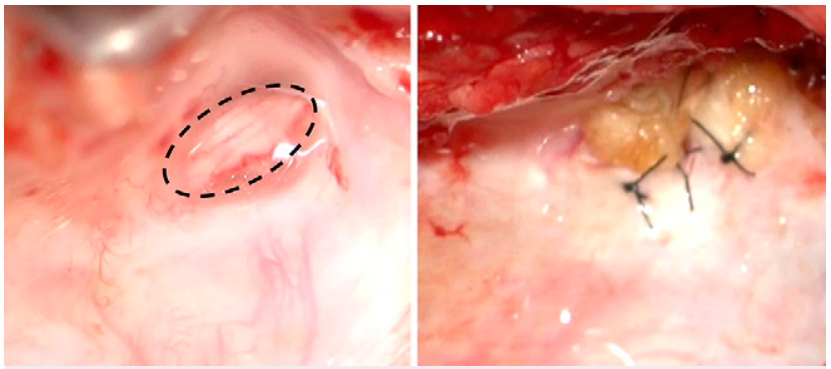

FIG. 3. Operative images of CSF fistula repair. Left: A posterior CSF fistula (surrounded by the black dotted line) without nerve root entrapment was identified. Right: The fistula was repaired using 5-0 monofilament polypropylene sutures with the fascia of the paravertebral muscle.

identified (Fig. 3, left) and subsequently repaired using 5-0 monofilament polypropylene sutures with the fascia of the paravertebral muscle because direct suturing could not be achieved due to hard dural tissue (Fig. 3, right). The dural closure was augmented with fibrin sealant application, followed by drain insertion and layered closure.

Postoperative lumbar MRI indicated complete excision of the pseudomeningocele with minimal CSF leakage (Fig. 4). Subsequently, the patient's dementia symptoms improved slightly, and she was transferred to another hospital to undergo ventriculoperitoneal (VP) shunt implantation-a system for draining CSF from the lateral ventricles to the peritoneal cavity. Ventricular enlargement was reduced after the implantation of the VP shunt (Fig. 5), and the dementia symptoms and gait disturbance dramatically improved.

\section{Discussion}

\section{Observations}

The incidence of pseudomeningocele and CSF fistula is unclear because many patients tend to be asymptomatic. Previous studies have reported an estimated prevalence of $0.068 \%-2 \% .^{7-10}$
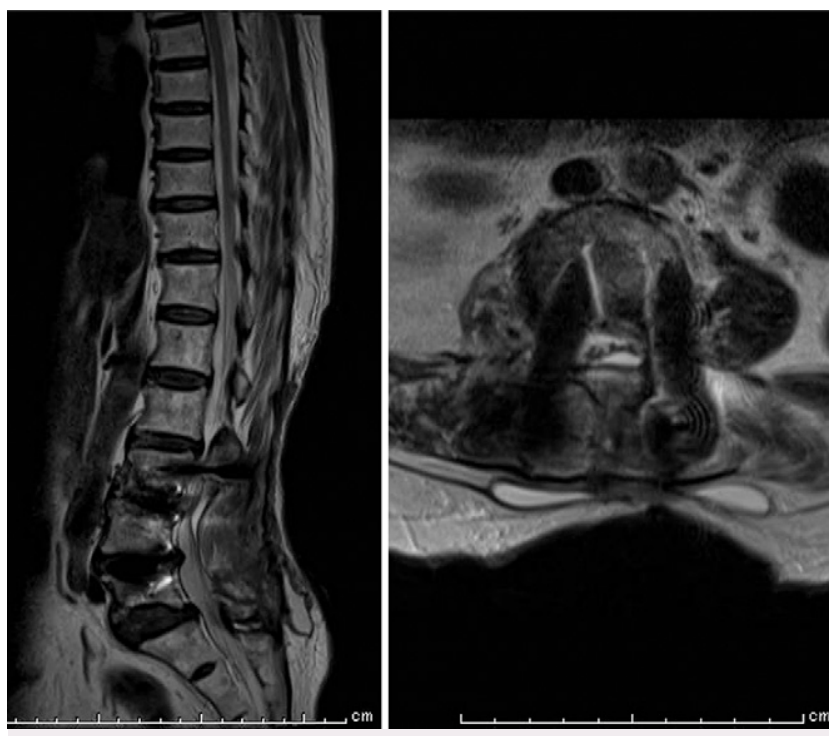

FIG. 4. Lumbar MRI after surgery for pseudomeningocele excision and dural repair. Left: T2-weighted sagittal image. Right: T2-weighted axial image. The pseudomeningocele was completely excised with minimal CSF leakage. 


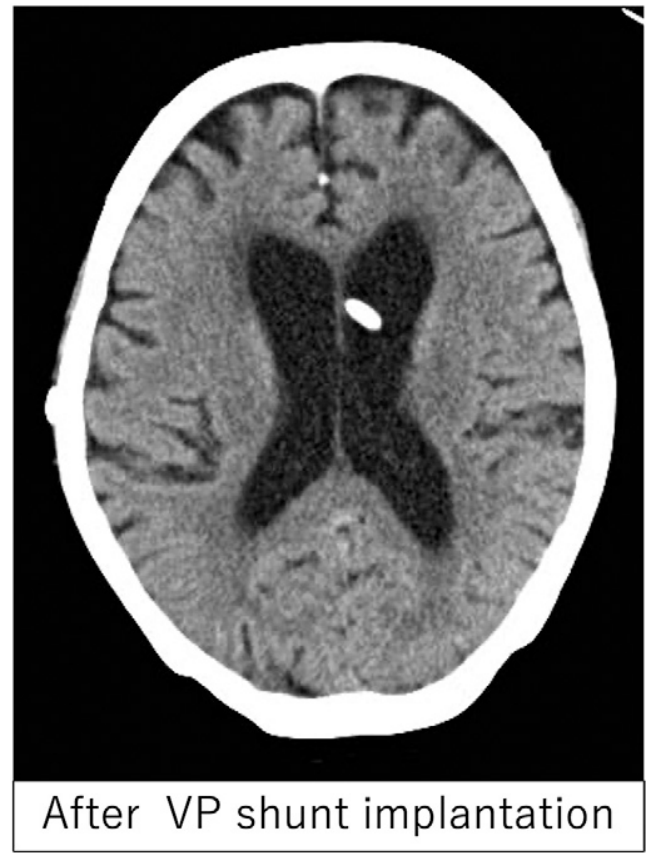

FIG. 5. Brain CT after VP shunt implantation. After the implantation of the VP shunt, the ventricular enlargement was reduced.

Dural tear is a relatively common complication after spine surgery. In primary surgery, the prevalence ranges from $3 \%$ to $5 \%{ }^{11}$ indicating that most dural tears or pseudomeningoceles are selfresolving and asymptomatic. Once a dural tear is identified, it should be appropriately assessed and treated. Failure to treat it could lead to the development of pseudomeningoceles, causing some symptoms, including headache, radiculopathy, and low back pain. Moreover, a dural tear in both the dural and arachnoid layers can lead to CSF leakage into the paraspinal tissues and development of a giant pseudomeningocele just under the skin, which can easily become infected.

For asymptomatic cases, it may be possible to wait for the spontaneous resolution of the pseudomeningoceles; Solomon et al. reported cases with spontaneous healing of the dural defect with gradual resorption of the CSF. ${ }^{12}$ Alternatively, for symptomatic cases, surgery for lumbar pseudomeningoceles may require careful closure of the dural tear with the nerve roots back into the dural sac.

$\mathrm{NPH}$ can be divided into two subtypes-one is idiopathic NPH, where the underlying cause is not previously known, and the second is secondary NPH, which is a result of various pathologies, such as subarachnoid hemorrhage, trauma, meningitis, stroke, or intracerebral hemorrhage. Secondary NPH can also be caused by fibrosis and adhesions in the subarachnoid space and arachnoid granulations. ${ }^{13,14}$ Although NPH cannot be resolved, many patients find relief through the surgical placement of a VP shunt. Therefore, the patient in this study was transferred to another hospital to undergo VP shunt implantation.

A tap test comprising $30-50 \mathrm{ml}$ of CSF drainage from patients followed by the assessment of symptom status is often used to determine the prognosis of NPH. ${ }^{15}$ However, in the present case, the pseudomeningocele was so large that we could not drain the real CSF inside the dura mater even after the puncture of the giant pseudomeningocele. Therefore, the diagnosis of NPH was not validated, which is a limitation of this study.

The association of CSF leakage with NPH has not been reported. However, the symptoms of NPH in the present case occurred after spinal surgery and recovered after dural repair surgery, indicating that the changes in CSF circulation and/or pressure due to pseudomeningoceles may cause NPH. Altered CSF dynamics may have induced the overproduction of CSF or may have induced the adhesions in the subarachnoid space of the brain, resulting in secondary NPH. Another possibility is that this patient had undiscovered $\mathrm{NPH}$, which may have delayed the onset of NPH symptoms by reducing pressure in the cerebrospinal cord due to postoperative pseudomeningocele.

Thus, the stabilization of CSF circulation and/or pressure through dural repair might lead to a reduction in the NPH symptoms, including dementia.

\section{Lessons}

The incidence of pseudomeningocele following spinal surgery is rare, and its occurrence could lead to the development of NPH. The prevention of dural tears through precise surgical technique and the primary repair of dural tears are the best approaches to prevent pseudomeningocele incidence and subsequent events.

\section{Acknowledgments}

We are grateful to the patient in this case report.

\section{References}

1. Enke O, Dannaway J, Tait M, New CH. Giant lumbar pseudomeningocele after revision lumbar laminectomy: a case report and review of the literature. Spinal Cord Ser Cases. 2018;4:82.

2. Hyndman OR, Gerber WF. Spinal extradural cysts, congenital and acquired; report of cases. J Neurosurg. 1946;3(6):474-486.

3. Cook DA, Heiner JP, Breed AL. Pseudomeningocele following spinal fracture. A case report and review of the literature. Clin Orthop Relat Res. 1989;(247):74-79.

4. Mayfield FH. Complications of laminectomy. Clin Neurosurg. 1976;23:435-439.

5. Lee KS, Hardy IM 2nd. Postlaminectomy lumbar pseudomeningocele: report of four cases. Neurosurgery. 1992;30(1):111-114.

6. Hakim S, Adams RD. The special clinical problem of symptomatic hydrocephalus with normal cerebrospinal fluid pressure. Observations on cerebrospinal fluid hydrodynamics. J Neurol Sci. 1965;2(4): 307-327.

7. Swanson HS, Fincher EF. Extradural arachnoidal cysts of traumatic origin. J Neurosurg. 1947;4(6):530-538.

8. Ahn Y, Lee HY, Lee SH, Lee JH. Dural tears in percutaneous endoscopic lumbar discectomy. Eur Spine J. 2011;20(1):58-64.

9. Teplick JG, Peyster RG, Teplick SK, Goodman LR, Haskin ME. CT identification of postlaminectomy pseudomeningocele. AJR Am J Roentgenol. 1983;140(6):1203-1206.

10. Khazim R, Dannawi Z, Spacey K, et al. Incidence and treatment of delayed symptoms of CSF leak following lumbar spinal surgery. Eur Spine J. 2015;24(9):2069-2076.

11. Papavero L, Engler N, Kothe R. Incidental durotomy in spine surgery: first aid in ten steps. Eur Spine J. 2015;24(9):2077-2084.

12. Solomon P, Sekharappa V, Krishnan V, David KS. Spontaneous resolution of postoperative lumbar pseudomeningoceles: a report of four cases. Indian J Orthop. 2013;47(4):417-421.

13. Daou B, Klinge P, Tjoumakaris $S$, Rosenwasser RH, Jabbour $P$. Revisiting secondary normal pressure hydrocephalus: does it exist? A review. Neurosurg Focus. 2016;41(3):E6. 
14. Oliveira LM, Nitrini R, Román GC. Normal-pressure hydrocephalus: a critical review. Dement Neuropsychol. 2019;13(2):133-143.

15. Gallagher R, Marquez J, Osmotherly P. Clinimetric properties and minimal clinically important differences for a battery of gait, balance, and cognitive examinations for the tap test in idiopathic normal pressure hydrocephalus. Neurosurgery. 2019;84(6):E378-E384.

\section{Disclosures}

The authors report no conflict of interest concerning the materials or methods used in this study or the findings specified in this paper.

\section{Author Contributions}

Conception and design: Okubo, Yato. Acquisition of data: Okubo, Yanai, Yato. Analysis and interpretation of data: Yato. Drafting the article:
Kobayashi, Yato. Critically revising the article: Takemitsu, Yato. Reviewed submitted version of manuscript: Kobayashi, Fujiyoshi, Shimizu, Kawano, Kato, Matsubayahi, Matsukawa, Furukawa, Konomi, Yamane, Yato. Approved the final version of the manuscript on behalf of all authors: Kobayashi. Statistical analysis: Yato. Administrative/technical/material support: Takemitsu, Yato. Study supervision: Yamane, Yato.

\section{Correspondence}

Yoshiomi Kobayashi: National Hospital Organization Murayama Medical Center, Tokyo, Japan. ykoba77@me.com. 